\title{
AN ANALYSIS OF MOBILE IPV6 SIGNALING LOAD IN NEXT GENERATION MOBILE NETWORKS
}

\author{
Sandro Grech ${ }^{1}$, Javier Poncela ${ }^{2}$, Pedro Serna ${ }^{1}$ \\ 1 Nokia Networks, P.O. Box 301, FIN-00045 Nokia Group, Finland \\ (sandro.grech@nokia.com; pedro.serna@nokia.com). \\ ${ }^{2}$ Department of Ingenieria de Comunicaciones, Universidad de Málaga, Málaga, 29071 \\ Spain(javier@ic.uma.es).
}

\begin{abstract}
To date, mobile communication has been dominated by voice services. This is likely to be valid also for the foreseeable future, but at the same time a multitude of data services are also emerging. This trend in mobile communication has fueled the introduction of packet-switched mobile networks, thus introducing the IP suite into the field of mobile communications. This technological shift can be already observed in today's 2.5 and $3 \mathrm{G}$ networks. In the first phases, however, mobile devices have an IP point of attachment which seldom changes throughout the lifetime of a communication session. Mobility management is handled below this point of attachment by means of access-specific mechanisms. A unified mobility management mechanism at the IP layer may enable streamlined network architectures, for example as complementary access technologies emerge in next generation mobile networks. Mobile IPv6 represents a key candidate mechanism to fulfill this vision of unified IP-based mobile communication networks. This paper analyses and quantifies the signaling overheads in a mobile communication network that uses Mobile IPv6 for mobility management.
\end{abstract}

Key words: IP signaling; Mobile IPv6; signaling load analysis; localized mobility management.

\section{INTRODUCTION}

IP is one of the key enablers of the anticipated widespread adoption of mobile multimedia and data services. The challenges that arise in this new 
environment are rather different from the ones that IP has traditionally faced. The main issue is related to mobility, which is inherent to wireless and cellular systems. Although mobility may be transparently handled using access-specific mechanisms, mobility may require the mobile device to change its IP address in order to maintain IP connectivity. Without Mobile IP, this change in IP address is exposed to the layers above IP, resulting in disruptions in the mobile communication. The analysis in this paper is specific to Mobile IPv6 [1], since IPv6 is assumed as an underlying enabler of widespread deployment of IP-based mobile communication.

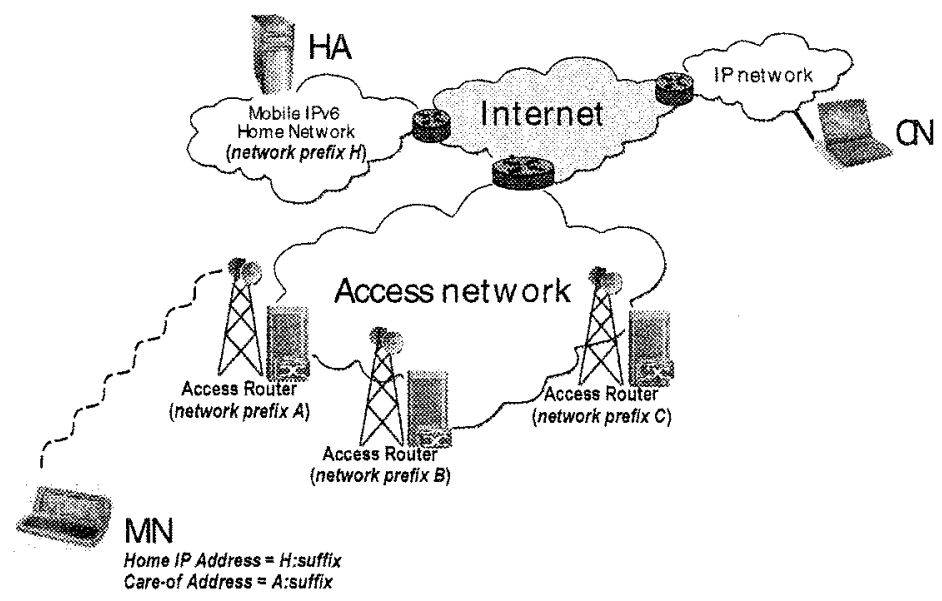

Figure 1. High level reference architecture.

In order to introduce mobility support in IPv6, Mobile IPv6 introduces several new concepts, which will be outlined next with reference to Figure 1. The Mobile Node (MN) acquires a static (or semi-static) IP address, known as the Home Address, from its Home Network. When the Mobile Node resides away from its Home Network it also acquires a Care-of Address $(\mathrm{CoA})$ that matches the prefix of the visited link. The Home Agent (HA) is a router on the home network that maintains a mapping (called binding cache entry) of the Mobile Node's Home Address with the Mobile Node's current Care-of Address. Correspondent Nodes (CN) are any nodes with which a Mobile Node is communicating. The Access Router (AR) is the router that the Mobile Node uses to obtain IP connectivity to the network. In this paper, it is assumed that IP base stations implement the AR functionality.

When a Mobile Node changes its network point of attachment it will require an IP address that matches the network prefix of the visited link. In order to maintain its reachability, the Mobile Node needs to announce this IP 
address to its Home Agent so that packets which reach the Mobile Node's home IP address can be re-directed by the Home Agent. The Mobile Node should also announce its Care-of Address to any Correspondent Nodes so that they can deliver the packets directly to the Mobile Node (this process is known as Route Optimization). These announcements are performed using messages known as Binding Updates (BU), which create a binding between a Mobile Node's Home Address and its Care-of Address at the receiving node. The bindings created by these messages expire after a pre-defined lifetime.

A Mobile Node announces its Care-of Address to the Home Agent and preferably also to its Correspondent Nodes in the following occasions:

a) a Mobile Node changes its Care-of Address,

b) the lifetime of an existing binding is about to expire (this can be triggered by a Correspondent Node by sending a Binding Refresh Request message),

c) additionally, a Mobile Node should also announce its Care-of Address to its Correspondent Nodes when a Mobile Node starts to communicate with a new Correspondent Node that is not aware of the Mobile Node's Care-of Address.

This study focuses on the Mobile IPv6 signaling load which is generated by a) in the list above. It assumes that the binding lifetimes (b) are long enough so that the signaling load caused in refreshing these bindings is negligibly low when compared to the load caused by IP address changes. The Mobile IPv6 signaling due to c) may or may not be substantial depending mainly on what type of applications the terminal is engaged in. During some web-browsing sessions, for example, the user may be following a set of hyperlinks that point to different web servers (and thus different Correspondent Nodes). In this example the Mobile IPv6 signaling due to change of Correspondent Node may be higher than the Mobile IPv6 signaling due to mobility. In some other applications, such as a speech call, the Correspondent Node is unique for the duration of the call. The overall result depends on the traffic mix, and on other application/user characteristics such as how often does a user using a web-browsing application follow a hyperlink that points to a new web server, etc. The signaling resulting from $\mathrm{c}$ ) is not considered in the calculations in this study.

\section{BINDING UPDATE PROCEDURE}

The Binding Update procedure is a core component of Mobile IPv6. This procedure is used by the Mobile Node to inform the Home Agent and any 
Correspondent Nodes that a new Care-of Address has been assigned to the Mobile Node.

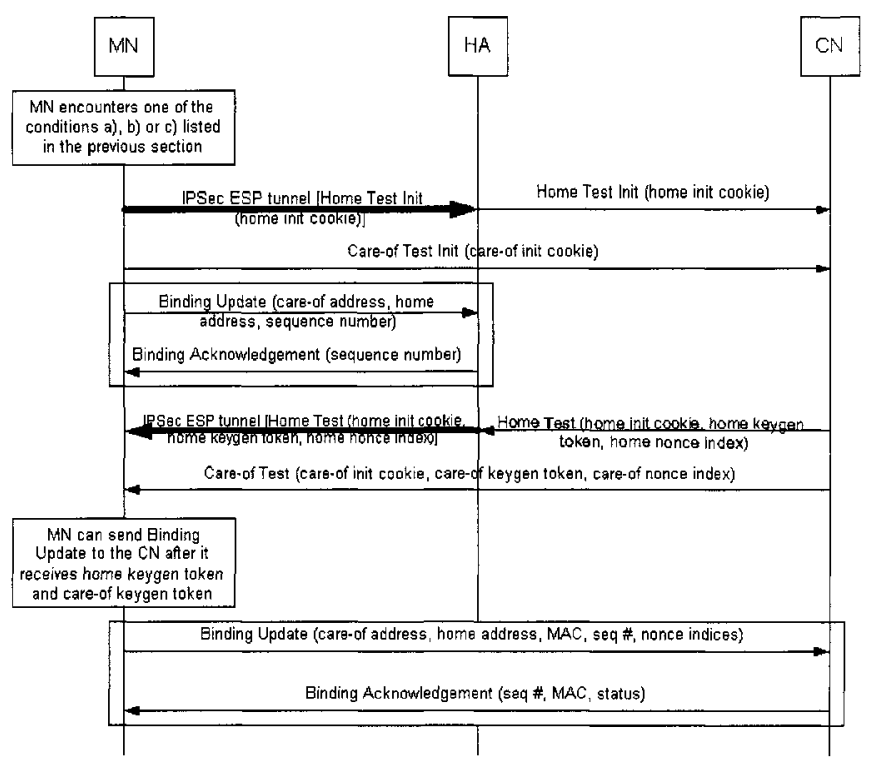

Figure 2. Mobile IPv6 Binding Update signaling.

The sequence of messages exchanged between Mobile Node, Home Agent and Correspondent Node when a Binding Update is required is illustrated in Figure 2. First, the Mobile Node sends two challenges to the Correspondent Node, one of them (Home Init cookie) secured via the Home Agent and the other (Care-of Init cookie), unprotected, directly to the Correspondent Node; these messages are known as Home Test Init (HoTI) and Care-of Test Init (CoTI), respectively. The Mobile Node can perform the binding procedure with the Home Agent in parallel to this operation.

The Mobile Node can send a valid Binding Update message to the Correspondent Node only after receiving two tokens (home and care-of keygen tokens) from the Correspondent Node in response to the Home Test Init and Care-of Test Init messages. The Mobile Node combines these tokens in order to generate the Message Authentication Code (MAC), which the Correspondent Node uses to validate the Binding Update. This procedure is called Return Routability. It enables the Correspondent Node to obtain some reasonable assurance that the Mobile Node is in fact addressable at its claimed Care-of Address and at its Home Address, before accepting any Binding Update from a Mobile Node. 


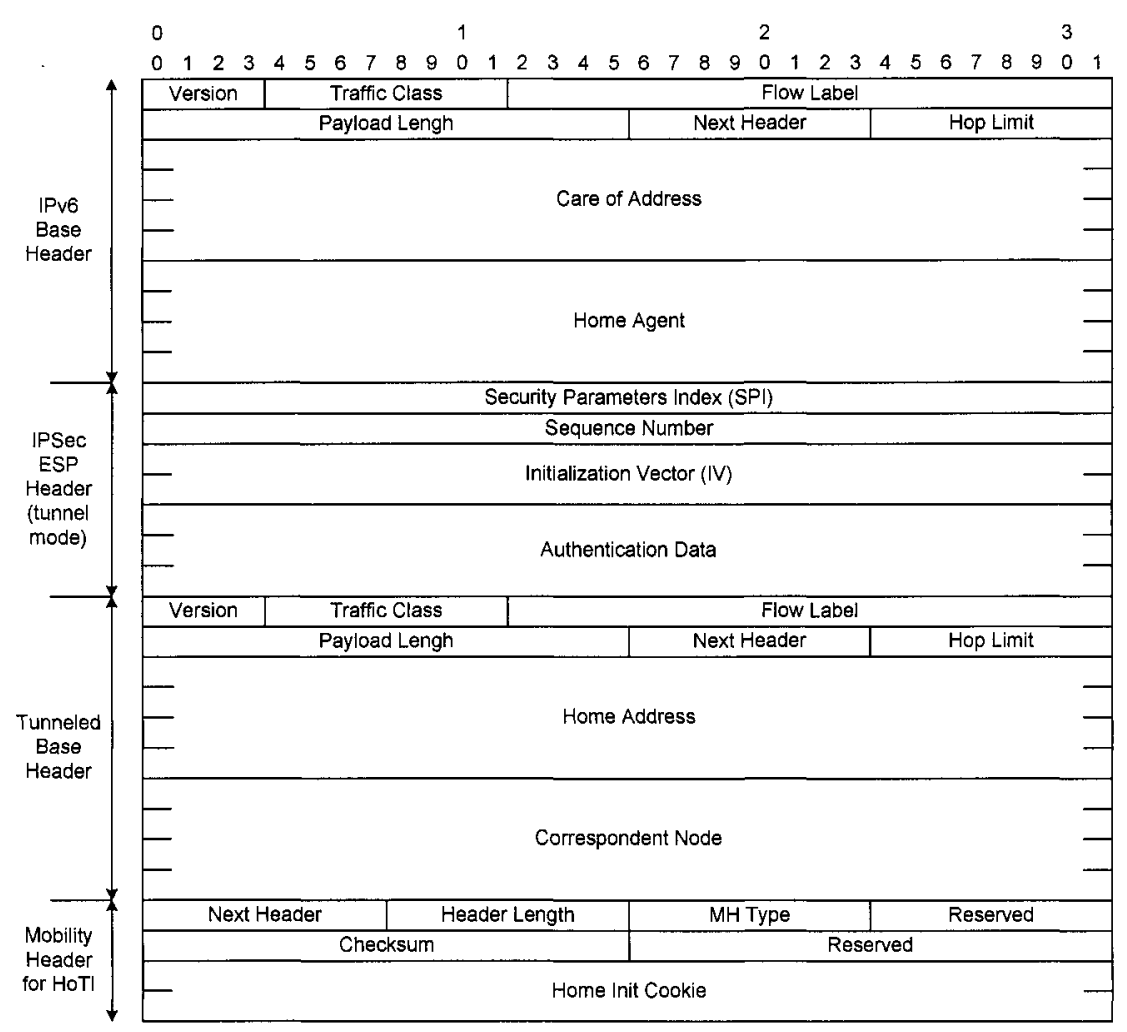

Figure 3. Format of message Home Test Init (HoTI).

The format of message Home Test Init (HoTI) sent from the Mobile Node to the Correspondent Node through the Home Agent (using tunneling) is shown in Figure 3. It is composed of an IPv6 header and a security header that encapsulates the message directed to the Correspondent Node. The source and destination addresses in the base header may be the Home Agent address, the Correspondent Node address or the Care-of Address, depending on the type of message [2]. The encapsulated message consists of an IPv6 header plus a mobility header that carries the Home Test Init message. Note that the 'Next Header' field in the Mobility header is named 'Payload Protocol' in [1].

The IPsec ESP header is used only for messages between the Mobile Node and the Home Agent. For all messages the format is similar but each message type will include its own fields after the Checksum of the Mobility Header and thus its size may vary [1]. Taking into account these variations, the size, in bytes, of the messages used in Figure 2 is provided in Table 1. The Initialization Vector and the Authentication Data fields depend on the Security Association [2]. 
Table 1. Mobile IPv6 Message Sizes

\begin{tabular}{lcccc}
\hline Message & Fixed Headers & IPsec & Message Data & Total Size \\
\hline Home Test Init (HoTI) & & & & \\
$\quad(M N \rightarrow H A)$ & 46 & 72 & 10 & 128 \\
$\quad(H A \rightarrow C N)$ & 46 & --- & 10 & 56 \\
Home Test $(H o T)$ & & & & \\
$\quad(C N \rightarrow H A)$ & 46 & --- & 18 & 64 \\
$\quad(H A \rightarrow M N)$ & 46 & 72 & 18 & 136 \\
Care-of Test Init (CoTI) & 46 & --- & 10 & 56 \\
Care-of Test & 46 & -- & 18 & 64 \\
Binding Update (BU) & & & & \\
$\quad(M N \rightarrow H A)$ & 46 & 72 & $18^{\prime}$ & 136 \\
$\quad(M N \rightarrow C N)$ & 46 & -- & $26^{2}$ & 72 \\
Binding Acknowledgement (BAck) & & & & \\
$\quad(H A \rightarrow M N)$ & 46 & 72 & 10 & 128 \\
$(C N \rightarrow M N)$ & 46 & --- & $26^{2}$ & 72 \\
\hline
\end{tabular}

${ }^{1}$ With no options.

${ }^{2}$ Assume Binding Authorization Data Option.

\section{BASELINE MOBILE IPV6 SIGNALING LOAD}

The signaling load due to Mobile IPv6 includes the load at the air interface, the load at the Home Agent and the load at the Correspondent Nodes. For the analysis, it has been assumed that no IP header compression is used for the Mobile IPv6 signaling packets. Also, it has been assumed that the home and care-of keygen tokens expire before the Mobile Node sends the next Binding Update to the same Correspondent Node. Due to this, the Mobile Node must perform the complete Binding Update procedure every time, going through the 'Home Test Init - Home Test' and 'Care of Test Init - Care of Test' exchanges every time that a Binding Update needs to be sent. The above assumptions clearly represent a worst-case scenario.

Table 2. Subscriber Density and Subscriber Mobility Figures

\begin{tabular}{cccc}
\hline Environment & AR relocations $/ 1000$ & Subscribers/AR & Relocations/second/AR \\
& subs/second $\left(R_{S}\right)$ & $\left(S_{C}\right)$ & $\left(R_{A R}\right)$ \\
\hline Urban & 0.556 & 339 & 0.189 \\
Dense Urban & 0.500 & 241 & 0.120 \\
Suburban & 0.476 & 1442 & 0.685 \\
\hline
\end{tabular}

The subscriber density and subscriber mobility parameters, which model the network, are shown in Table 2. Environments are classified depending on the cell size, which is smaller in dense urban (approx. $0.25 \mathrm{~km}^{2}$ ) and larger in a suburban environment (approx. $7 \mathrm{~km}^{2}$ ). These reference scenarios are based on typical values for density of data users utilized in equivalent cellular reference models. 
The air interface signaling overhead per AR can be obtained from the messages shown in Figure 2. The overhead in the uplink direction is the size of the messages (HoTI, CoTI, and both Binding Updates) sent by a Mobile Node due to an AR relocation, multiplied by the number of relocations per second per $A R\left(R_{A R}\right)$; for the downlink direction the calculation is similar.

The total size of the messages exchanged in the air interface (see Figure 2) is: $128(\mathrm{HoTI})+56(\mathrm{CoTI})+136(\mathrm{BU}$ to $\mathrm{HA})+72(\mathrm{BU}$ to $\mathrm{CN})+136$ $(\mathrm{HoT})+64(\mathrm{CoT})+128(\mathrm{BAck}$ from HA $)+72(\mathrm{BAck}$ from $\mathrm{CN})=792$ bytes. Thus, the total overhead per Access Router for an urban environment would be 149.69 bytes $/ \mathrm{s}(\approx 1.2 \mathrm{kbit} / \mathrm{s} / \mathrm{AR})$, which represents around $0.6 \mathrm{kbit} / \mathrm{s}$ in each direction. This overhead is negligible, even considering bandwidthlimited interfaces.

The air interface signaling load for various environments is shown in Figure 4.a. For suburban ARs the amount of Mobile IPv6 signaling overhead tends to be higher. This is mainly due to the high number of Mobile Nodes per AR in the suburban area (1442 MNs/AR). Results show that, even with multiple simultaneous $\mathrm{CNs}$, the air interface signaling overhead per AR is not substantial.

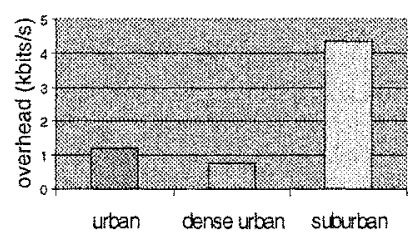

(a) Air interface signaling overhead per AR.

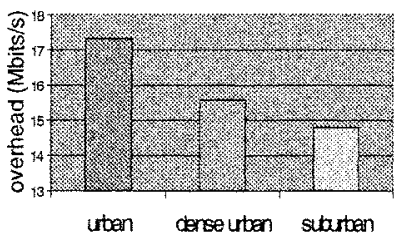

(c) Signaling overhead at Home Agent for 6 million subscribers.

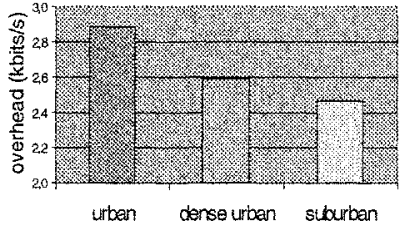

(b) Signaling overhead at Home Agent per 1000 subscribers.

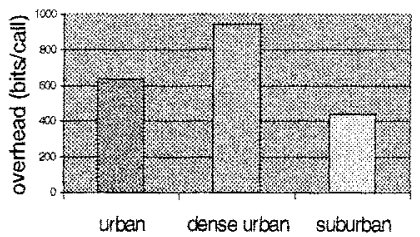

(d) Signaling overhead at Correspondent Node per Mobile Node per call.

Figure 4. Baseline Mobile IPv6 signaling overheads.

The load at the Home Agent is given by the size of the messages involved in the Home Agent signaling: 128 (HoTI) $+64(\mathrm{HoT})+136$ (BU to $\mathrm{HA})+56(\mathrm{HoTI})+136(\mathrm{HoT})+128($ BAck from HA) $=648$ bytes. To obtain the total number of bytes which are processed (either received or sent) by the Home Agent, this number must be multiplied by the number of subscribers served by the Home Agent $(\mathrm{N})$ and the number of relocations per 
1000 subscribers per second: $648 \times \mathrm{N} \mathrm{x} \mathrm{R}_{\mathrm{s}}$. In an urban environment, the signaling overhead at the Home Agent per 1000 subscribers would be 360 bytes/s $(\approx 2.89 \mathrm{kbit} / \mathrm{s})$ (see Figure $4 . \mathrm{b}$ for estimations on various environments).

Moreover, the processing overhead of a Mobile IPv6 BU-BAck pair has been measured [4] to be approximately as reported in Table 3. The Home Agent must also initiate and terminate the IPsec ESP tunnel to the Mobile Node. The additional processing can be roughly estimated to be double. From these results, one can infer that a Home Agent based on a $1 \mathrm{GHz}$ processor can process approximately 5,000 'Binding Update - Binding Acknowledgement' pairs per second.

Table 3. Overhead of a BU-BAck Message Pair in the Home Agent

\begin{tabular}{lcc}
\hline & No Piggyback & Piggybacked \\
\hline Sending cost & $0.20 \mathrm{~ms}$ & $0.23 \mathrm{~ms}$ \\
Receiving cost & $0.29 \mathrm{~ms}$ & $0.23 \mathrm{~ms}$ \\
\hline
\end{tabular}

A Home Agent serving $6 \times 10^{6}$ subscribers would need to process approximately 3,300 'Binding Update - Binding Acknowledgement' pairs per second. This means that, in theory, if a Home Agent processor does not handle any other traffic, current state of the art processors easily manage this amount of processing [4]. The signaling overhead at a Home Agent serving 6 million mobile subscribers is shown in Figure 4.c. This shows that the bandwidth introduced by 6 million Mobile IPv6 MNs at the Home Agent could be easily supported on a $100 \mathrm{Mbit} / \mathrm{s}$ interface.Mobile Nodes should also send Binding Updates to any Correspondent Nodes with which they have ongoing communication, in order to facilitate the operation of route optimization for Mobile IPv6. For a Correspondent Node the overhead caused by the binding messages for this server would be given by the equation:

$$
O H_{B U}=\frac{\left(S_{B U}+S_{C O T I}+S_{H o T I}+S_{C O T}+S_{H o T}+S_{B \backslash c k}\right) \times R_{A N E}}{R_{u} \times\left(S_{d}+S_{u}\right)}
$$

where $S_{d}$ is the average downlink payload size is, is $S_{u}$ is the average uplink payload size, $R_{u}$ is the average uplink packet rate and $R_{M N}$ is the average AR relocations/second/MN. Taking a voice service as an example and using the values given in Table 4 , the signaling overhead at the Correspondent Node would be $O H_{B U}=0.196 \times R_{M N}$.

For voice calls, assuming an average duration of 90 seconds we obtain a mean value of 2.46 relocations/call. Thus, $\mathrm{R}_{\mathrm{MN}}=2.46 / 90$, which produces 0.027 relocations per second per Mobile Node in a dense urban environment. This amounts to an overhead due to Binding Updates of 945 bits per call, which turns to be less than $0.6 \%$ overhead in the Correspondent Node per 
Mobile Node, relative to user bandwidth. Figure 4.d shows the signaling overhead at the Correspondent Node per call for the different environments.

Table 4. Typical Traffic Characteristics

\begin{tabular}{lccccc}
\hline & Voice & Video & Streaming & $\begin{array}{c}\text { Web- } \\
\text { browsing }\end{array}$ & $\begin{array}{c}\text { File } \\
\text { transfer }\end{array}$ \\
\hline Packtes/second/direction $\left(R_{u}\right)$ & 32.5 & 30 & 6 & 25 & 50 \\
Mean payload length uplink $\left(S_{u}\right)($ bytes) & 30.1 & 250 & 25 & 125 & 200 \\
Mean payload length downlink $\left(S_{d}\right)$ & 30.1 & 250 & 500 & 500 & 800 \\
(bytes) & 7.8 & 60 & 24 & 50 & 80 \\
Average User Bitrate (downlink) (kbps) & 7.8 & 60 & 12 & 12.5 & 20 \\
Average User Bitrate (uplink) (kbps) & & & & &
\end{tabular}

As can be seen from the results presented, the signaling load and processing overhead introduced by Mobile IPv6 are not critical for any of the affected parties, i.e. the air interface, the Home Agent, and the Correspondent Node. Results show that systems could cope with this overhead with slight increases of their actual capacity, which should be considered in the planning stage. Thus, the introduction of Mobile IPv6 is not hindered by the overheads studied in this section.

\section{ANALYSIS OF INBAND SIGNALING}

Apart from signaling due to specific messages required by Mobile IPv6, overheads must also consider the additional signaling included in user packets due to Mobile IP. There are four components that must be considered: Home Address Destination option, Routing Header, inband signaling when the Correspondent Node is also a Mobile Node and IP header compression; their analysis is provided below. For this analysis, traffic estimations per user are required, and the results are dependent on the specific mix of traffic assigned to one user.

In order to avoid ingress filtering of packets by the Access Router, the Mobile Node uses its Care-of Address as IP Source Address when sending packets (uplink). To make the use of the Care-of Address transparent to the higher layers (above IP), each packet must also include a 20 byte Home Address Destination Option. This destination option contains the Home Address of the Mobile Node, which will be used by the Correspondent Node to replace the Care-of Address in the IP Source Address field before being passed to the higher layers.

The overhead associated with the Home Address Destination Option is represented in Table 5(a). For example, for voice service, as there are on average 30.1 bytes per packet, adding 20 bytes for the Home Address 
Destination Option would represent an overhead of $66 \%$ relative to the payload. If we include the size of the headers (around 60 bytes), without compression mechanisms, the overhead goes down to $21.7 \%$. As there are 32.5 packets per second in each direction this amounts to around $5 \mathrm{kbps}$ per voice flow in the uplink direction. The average subscriber uplink bitrate in the Busy Hour, due to the combined use of all services, experiences an increase from $0.818 \mathrm{kbps}$ to $0.962 \mathrm{kbps}$. This represents an increase of $17 \%$ in uplink network traffic.

Table 5. Mobile IPv6 overhead in (a) uplink traffic due to Home Address Destination Option and (b) downlink traffic due to Routing Header

\begin{tabular}{lcccccc}
\hline & $\begin{array}{c}\text { Overhead relative to } \\
\text { payload (\%) }\end{array}$ & $\begin{array}{c}\text { Overhead (with headers) } \\
(\%)\end{array}$ & \multicolumn{2}{c}{$\begin{array}{c}\text { Overhead per flow } \\
\text { (kbps) }\end{array}$} \\
\hline & $($ a $)$ & $(b)$ & $(a)$ & $(b)$ & $(a)$ & $(b)$ \\
\hline Voice & 66 & 80.0 & 21.7 & 26.0 & 5.2 & 6.2 \\
Video & 8 & 9.6 & 6.1 & 7.4 & 4.8 & 5.8 \\
Streaming & 80 & 4.8 & 20.2 & 4.1 & 1 & 1.1 \\
Web-browsing & 16 & 4.8 & 10.5 & 4.2 & 4 & 4.8 \\
File-transfer & 10 & 3.0 & 4.3 & 2.7 & 8 & 9.6 \\
\hline
\end{tabular}

In the downlink direction, packets sent from the $\mathrm{CN}$ to the $\mathrm{MN}$ carry the Mobile Node's Care-of Address in the Destination Address field of the IPv6 header. Mobile IPv6 uses a 24 byte Routing Header (type 2) to carry the Mobile Node's Home Address in every packet. This enables the Mobile Node to replace its CoA in the IPv6 header with the Home Address in the Routing Header before handing the packet over to the layers above IP. These higher layers are therefore only aware of the Mobile Node's static Home Address.

The overhead associated with the Routing Header is represented in Table 5(b). In this case, downlink traffic parameters are concerned. For example, for voice service, adding 24 bytes for the Routing Header Option would represent an overhead of $80 \%$ relative to the payload. If we include the size of the headers, the relative overhead goes down to $26 \%$. This amounts to slightly more than $6 \mathrm{kbps}$ per voice flow in the downlink direction. The average subscriber downlink bitrate, due to the combined use of all services, in the Busy Hour experiences an increase, due to the use of the Routing Header Option, from $1.14 \mathrm{kbps}$ to $1.31 \mathrm{kbps}$. This represents an increase of $15 \%$ in downlink network traffic.

If the Correspondent Node is also a Mobile Node using Mobile IPv6, then each packet between the two nodes needs both a Routing Header and a Home Address Destination Option. This translates to 44 bytes of overhead in every packet between the two nodes. If we assume that $\mathrm{MN}$ to $\mathrm{MN}$ communication will be either voice or video (see Table 4 for typical packet 
sizes) Mobile IPv6 causes an overhead of around 25\% in the uplink network traffic and around $35 \%$ in the downlink network traffic.

Since the contents of the Home Address Destination Option and Type-2 Routing Header within a Route Optimized Mobile IPv6 user packet are fairly constant, it is expected that IP header compression algorithms, such as the IETF Robust Header Compression (ROHC) [5], will be capable of compressing these headers introduced to the user packets by Mobile IPv6. If IP header compression is applied over the air interface, the impact of the Mobile IPv6 headers will be minimized over this resource limited link. In the wired part of the network, however, the full headers will be exposed and thus the overheads calculated in the previous sections are valid for this part of the network.

\section{CONCLUSION}

The results of the baseline Mobile IPv6 signaling load show that the overhead caused by the introduction of Mobile IPv6 in next generation mobile systems is rather small. For example, the air interface signaling load per AR, with more than 300 subscribers, in an urban environment is around $1.2 \mathrm{kbit} / \mathrm{s}$. In the Correspondent Nodes the increase of load amounts to less than $1 \%$ per Mobile Node. Also, the additional signaling and processing loads in the Home Agent are also in reasonably low limits.

The inband signaling caused by Mobile IPv6 due to the use of the Home Address Destination Option in uplink messages and the Routing Header Option in downlink messages is, however, bigger, having its highest impact on voice services. The total overhead depends on the exact user traffic mix, increasing as the voice services represent a bigger share of the total traffic. Our results show that this overhead would result in an increase of more than $15 \%$ on the traffic, both in the uplink and downlink directions. The impact of this overhead could be reduced using header compression mechanisms in the air interface. If the Correspondent Node is also a Mobile Node using Mobile IPv6, then the overhead caused by Mobile IPv6 is around 25\% in the uplink direction and $35 \%$ in the downlink.

From the standpoint of mobility signaling, Mobile IPv6 scales well by maintaining sustainable levels of overhead at the AR, intra-domain links, Home Agent and Correspondent Nodes. This is mainly accredited to the relatively low relocation frequencies characteristic of cellular environments.

Several proposals for introducing hierarchical mobility to Mobile IP have appeared in the past years ([6], [7]), in order to address the issue of signaling load, signaling overhead, and handover speed in Mobile IPv6. These 
mechanisms are however aimed towards the reduction of signaling load and signaling overhead associated with the Mobile IPv6 outband signaling (i.e. BU, BAck, CoTI, HoTI, CoT and HoT). The analysis in this paper shows these are not so critical when compared to the inband signaling and user plane processing. If coupled with a mechanism such as [8], which reduces the Mobile IPv6 handover latency, we thus believe that a hierarchical extension to Mobile IPv6 is not necessary for the architecture considered in this paper.

\section{ACKNOWLEDGEMENTS}

This work was performed as part of a co-operation agreement between Nokia and the University of Málaga.

\section{REFERENCES}

1. D. B. Johnson, C. E. Perkins, "Mobility support in IPv6", RFC 3775.

2. J. Arkko, V. Devarapalli, F. Dupont, "Using IPsec to Protect Mobile IPv6 Signaling between Mobile Nodes and Home Agents", RFC 3776.

3. S. Kent, R. Atkinson, "IP Encapsulating Security Payload (ESP)" RFC 2406, November 1998.

4. R. Wakikawa, "The Design and Implementation of Mobile IPv6 with multiple network interface support", Master's Thesis, Keijo University - Japan, June 2000.

5. C. Bormann, et al., "Robust Header Compression (ROHC)", RFC 3095.

6. H. Soliman, C. Castelluccia, K. El-Malki, L. Bellier, "Hierarchical Mobile IPv6 Mobility Management (HMIPv6)", draft-ietf-mipshop-hmipv6-02.txt, June, 2004.

7. A. Campbell, J. Gomez, S. Kim, A. Valko and C.Y. Wan, "Design, Implementation, and Evaluation of Cellular IP", IEEE Personal Communications, pp. 42-49, August 2000.

8. R. Koodli, et al., "Fast Handovers for Mobile IPv6", draft-ietf-mipshop-fastmipv6-01.txt, January, 2004. 